Copyright (C) 2002 IFAC

15th Triennial World Congress, Barcelona, Spain

\title{
FEEDBACK CONTROL OF LINEAR MULTIPARAMETER SINGULARLY PERTURBED SYSTEMS
}

\author{
Hiroaki Mukaidani ${ }^{*}$ Hua $\mathrm{Xu}^{* *}$ Koich Mizukami ${ }^{* * *}$ \\ * Faculty of Information Sciences, Hiroshima City University, \\ 3-4-1, Ozukahigashi Asaminami-ku Hiroshima, 731-3194 Japan. \\ e-mail:mukaida@im.hiroshima-cu.ac.jp \\ ** Graduate School of Business Sciences, The University of \\ Tsukuba, 3-29-1, Otsuka Bunkyou-ku Tokyo, 112-0012 Japan. \\ *** Faculty of Engineering, Hiroshima Kokusai Gakuin \\ University, 6-20-1, Nakano Aki-ku Hiroshima, 739-0321 Japan.
}

\begin{abstract}
In this paper, the linear quadratic optimal control problem for multiparameter singularly perturbed systems (MSPS) is studied in a different approach from the existing methods. The attention is focused on the design of a near-optimal controller which does not depend on the values of the small unknown parameters. It is shown that the resulting controller achieves $O\left(\|\mu\|^{2}\right)$ approximation of the optimal cost for the special case of the fast subsystems compared with the existing results. Moreover, it is also shown that the resulting controller is equivalent to the existing composite controller.
\end{abstract}

Keywords: Multiparameter singularly perturbed systems (MSPS), Multiparameter algebraic Riccati equation (MARE), Linear quadratic optimal control problem, Nnear-optimal control

\section{INTRODUCTION}

The deterministic and stochastic multimodeling stability, control, filtering and dynamic games have been investigated extensively by several researchers (see e.g., Khalil and Kokotović, 1978, 1979; Coumarbatch and Gajić, 2000; Gajić, 1988; Wang et al., 1994). The multimodeling problems arise in large-scale dynamic systems. For example, these multimodel situations in practice are illustrated by the multiarea power system (Khalil and Kokotović, 1978). In order to obtain the optimal solution to the multimodeling problems, we must solve the multiparameter algebraic Riccati equation (MARE), which are parameterized by the small positive same order parameters $\varepsilon_{j}, j=$ $1,2, \cdots$ Various reliable approaches for solving the MARE have been well documented in literatures (see e.g., Coumarbatch and Gajić, 2000;
Mukaidani et al. 2002). However, these results are limited to the case that the small parameters are assumed to be known. Thus, it is not applicable to a large class of problems where the parameters represent small unknown perturbations whose values are not known exactly.

A popular approach to deal with the MSPS is the two-time-scale design method (see e.g., Khalil and Kokotović, 1978, 1979; Gajić, 1988; Kokotović et al., 1986). For example, optimal control of a class of the MSPS has been studied by Khalil and Kokotović (1979), where the design of the $\varepsilon_{j}$-independent reduced-order controller has been suggested. When $\varepsilon_{j}$ is very small or unknown the previously used technique is very efficient. In Wang et al. (1994), using the descriptor variable approach, the main results of Khalil and Kokotović (1979) have been improved for the nonstan- 
dard MSPS such that at least one of the fast state matrices is singular. However, the existing controllers proposed in Wang et al. (1994) and Khalil and Kokotović (1979) only achieve $O(\|\mu\|)$ (where $\left.\mu=\left[\begin{array}{ll}\varepsilon_{1} & \varepsilon_{2}\end{array}\right]\right)$ approximation of the optimal cost.

In this paper, we study the linear quadratic optimal control problem for the MSPS. For this purpose, we first investigate the unique and bounded solution of the MARE and establish its asymptotic structure. Using the asymptotic structure, a new near-optimal controller which does not depend on the values of the small parameters is obtained. This is done by eliminating the parameters $\varepsilon_{j}$ for the full-order controller. It should be pointed out that the design method proposed in this paper is quite different from the two-timescale design method and the descriptor variable approach (Wang et al., 1994). As a result, we have only to solve the algebraic Riccati equation (ARE) with same order dimension of the reducedorder slow and each fast systems which do not depend on the values of the small parameters. It is emphasized that the resulting controller achieves $O\left(\|\mu\|^{2}\right)$ approximation of the optimal cost for the special case of the fast subsystems compared with the previously proposed controller in (Khalil and Kokotović, 1979; Wang et al., 1994). Even if the parameters are unknown, when the parameters are sufficiently small, the proposed near-optimal controller can be used reliably for the MSPS. As another important feature, when the fast state matrix $A_{j j}$ is nonsingular, we show that the resulting controller is equivalent to the composite controller which is based on the two-time-scale design method. Therefore, we claim that the new near-optimal controller includes the existing one as a special case.

\section{THE MSPS}

We consider the linear time-invariant MSPS

$$
\begin{aligned}
\dot{x}_{0}(t)= & A_{00} x_{0}(t)+A_{01} x_{1}(t)+A_{02} x_{2}(t) \\
& +B_{01} u_{1}(t)+B_{02} u_{2}(t), x_{0}(0)=x_{0}^{0}, \\
\varepsilon_{1} \dot{x}_{1}(t)= & A_{10} x_{0}(t)+A_{11} x_{1}(t)+\varepsilon_{12} A_{12} x_{2}(t) \\
& +B_{11} u_{1}(t), x_{1}(0)=x_{1}^{0}, \\
\varepsilon_{2} \dot{x}_{2}(t)= & A_{20} x_{0}(t)+\varepsilon_{21} A_{21} x_{1}(t)+A_{22} x_{2}(t) \\
& +B_{22} u_{2}(t), x_{2}(0)=x_{2}^{0},
\end{aligned}
$$

where $x_{j} \in \mathbf{R}^{n_{j}}, j=0,1,2$ are the state vectors, $u_{j} \in \mathbf{R}^{m_{j}}, j=1,2$ are the control inputs. All the matrices are constant matrices of appropriate dimensions.

$\varepsilon_{1}$ and $\varepsilon_{2}$ are two small positive singular parameters of the same order of magnitude such that

$$
0<\underline{k}_{12} \leq \alpha \equiv \frac{\varepsilon_{1}}{\varepsilon_{2}} \leq \bar{k}_{12}<\infty .
$$

That is, we assume that the ratio of $\varepsilon_{1}$ and $\varepsilon_{2}$ is bounded by some positive constants $\underline{k}_{12}$ and $\bar{k}_{12}$. $\varepsilon_{12}$ and $\varepsilon_{21}$ are two weak coupling between the fast subsystems. Note that the coupling parameters $\varepsilon_{12}$ and $\varepsilon_{21}$ can be positive, negative or zero. We note that the fast state matrices $A_{j j}, j=1,2$ may be singular. In the optimal control of the above MSPS, the performance criterion is given by

$$
\begin{aligned}
J & =\frac{1}{2} \int_{0}^{\infty} z^{T}(t) z(t) d t, \\
z(t) & =C\left[\begin{array}{l}
x_{0}(t) \\
x_{1}(t) \\
x_{2}(t)
\end{array}\right]+D\left[\begin{array}{l}
u_{1}(t) \\
u_{2}(t)
\end{array}\right]=C x(t)+D u(t),
\end{aligned}
$$

where

$$
\begin{aligned}
& C:=\left[\begin{array}{ccc}
C_{10} & C_{11} & 0 \\
C_{20} & 0 & C_{22}
\end{array}\right], \\
& C^{T} C=Q:=\left[\begin{array}{ccc}
Q_{00} & Q_{01} & Q_{02} \\
Q_{01}^{T} & Q_{11} & 0 \\
Q_{02}^{T} & 0 & Q_{22}
\end{array}\right], \\
& D^{T} D=R:=\left[\begin{array}{cc}
R_{1} & 0 \\
0 & R_{2}
\end{array}\right]>0, C^{T} D=0 .
\end{aligned}
$$

It is well known that the solution of the linear quadratic control problem (1) and (3) is given by (Khalil and Kokotović, 1978; Coumarbatch and Gajić, 2000),

$$
u_{\mathrm{opt}}(t)=\left[\begin{array}{l}
u_{1}(t) \\
u_{2}(t)
\end{array}\right]=-R^{-1} B_{e}^{T} P_{e} x(t),
$$

where $P_{e}$ satisfies the MARE

$$
A_{e}^{T} P_{e}+P_{e} A_{e}-P_{e} S_{e} P_{e}+Q=0
$$

with

$$
\begin{aligned}
& A_{e}:=\left[\begin{array}{ccc}
A_{00} & A_{01} & A_{02} \\
\varepsilon_{1}^{-1} A_{10} & \varepsilon_{1}^{-1} A_{11} & \varepsilon_{1}^{-1} \varepsilon_{12} A_{12} \\
\varepsilon_{2}^{-1} A_{20} & \varepsilon_{2}^{-1} \varepsilon_{21} A_{21} & \varepsilon_{2}^{-1} A_{22}
\end{array}\right] \\
& \in \mathbf{R}^{\bar{n} \times \bar{n}}, \bar{n}:=n_{0}+n_{1}+n_{2} \text {, } \\
& S_{e}:=B_{e} R^{-1} B_{e}^{T}=\left[\begin{array}{ccc}
S_{00} & \varepsilon_{1}^{-1} S_{01} & \varepsilon_{2}^{-1} S_{02} \\
\varepsilon_{1}^{-1} S_{01}^{T} & \varepsilon_{1}^{-2} S_{11} & 0 \\
\varepsilon_{2}^{-1} S_{02}^{T} & 0 & \varepsilon_{2}^{-2} S_{22}
\end{array}\right] \\
& \in \mathbf{R}^{\bar{n} \times \bar{n}}, \\
& B_{e}:=\left[\begin{array}{cc}
B_{01} & B_{02} \\
\varepsilon_{1}^{-1} B_{11} & 0 \\
0 & \varepsilon_{2}^{-1} B_{22}
\end{array}\right] \\
& \in \mathbf{R}^{\bar{n} \times \bar{m}}, \bar{m}:=m_{1}+m_{2} \text {. }
\end{aligned}
$$

Moreover, the optimal cost is given by 


$$
J_{\mathrm{opt}}=\frac{1}{2} x(0)^{T} P_{e} x(0) .
$$

If we know the values of the small parameters $\varepsilon_{1}, \varepsilon_{2}, \varepsilon_{12}$ and $\varepsilon_{21}$, this optimal control problem could be solved (Coumarbatch and Gajić, 2000; Mukaidani et al. 2002). However, it is impossible to obtain the optimal control when the small parameters are unknown. In such cases, the exact controller (4) cannot be used.

A near-optimal control design for the MSPS has been proposed in Khalil and Kokotović (1978, 1979). The algorithm consists of solving three separate subproblems, one in a slow time scale and two in fast time scale, and then combining the solutions of these problems to form a composite controller. However, in order to separate the MSPS the nonsingularity of the matrices $A_{j j}, j=$ 1, 2 are required. To avoid these assumptions we propose a new design method, which is based on the approximate theory in a different approach from the composite design.

\section{THE MARE}

Before we present the near-optimal controller, we first introduce the asymptotic structure for the MARE (5). A solution $P_{e}$ of the MARE (5), if it exists, must contain the parameters $\varepsilon_{j}, j=1,2$ because the matrices $A_{e}$ and $B_{e}$ contain the $\varepsilon_{j}^{-1}$ order parameter. Taking into account this fact, we look for a solutions $P_{e}$ of the MARE (5) with the structure

$$
P_{e}:=\left[\begin{array}{ccc}
P_{00} & \varepsilon_{1} P_{10}^{T} & \varepsilon_{2} P_{20}^{T} \\
\varepsilon_{1} P_{10} & \varepsilon_{1} P_{11} & \sqrt{\varepsilon_{1} \varepsilon_{2}} P_{21}^{T} \\
\varepsilon_{2} P_{20} & \sqrt{\varepsilon_{1} \varepsilon_{2}} P_{21} & \varepsilon_{2} P_{22}
\end{array}\right] \in \mathbf{R}^{\bar{n} \times \bar{n}}(7)
$$

where $P_{00}=P_{00}^{T}, P_{11}=P_{11}^{T}, P_{22}=P_{22}^{T}$.

It is assumed that the limit of $\alpha$ exists as $\varepsilon_{1}$ and $\varepsilon_{2}$ tend to zero, that is (see e.g., Khalil and Kokotović, 1978, 1979)

$$
\bar{\alpha}=\lim _{\substack{\varepsilon_{1} \rightarrow+0 \\ \varepsilon_{2} \rightarrow+0}} \alpha .
$$

Furthermore, without loss of generality, the following assumptions are made (Wang et al. 1994).

Assumption 1: The triples $\left(A_{j j}, B_{j j}, C_{j j}\right), j=$ 1, 2 are stabilizable and detectable.

\section{Assumption 2:}

$$
\begin{array}{r}
\operatorname{rank}\left[\begin{array}{ccccc}
s I_{n_{0}}-A_{00} & -A_{01} & -A_{02} & B_{01} & B_{02} \\
-A_{10} & -A_{11} & 0 & B_{11} & 0 \\
-A_{20} & 0 & -A_{22} & 0 & B_{22}
\end{array}\right]=\bar{n} \\
\operatorname{rank}\left[\begin{array}{ccccc}
s I_{n_{0}}-A_{00}^{T} & -A_{10}^{T} & -A_{20}^{T} & C_{10}^{T} & C_{20}^{T} \\
-A_{01}^{T} & -A_{11}^{T} & 0 & C_{11}^{T} & 0 \\
-A_{02}^{T} & 0 & -A_{22}^{T} & 0 & C_{22}^{T}
\end{array}\right]=\bar{n}
\end{array}
$$

where $\operatorname{Re}[s] \geq 0, \quad s \in \mathrm{C}$.

Assumption 3: The Hamiltonian matrix $T_{j j}, j=$ 1,2 is nonsingular, where

$$
T_{j j}:=\left[\begin{array}{cc}
A_{j j} & -S_{j j} \\
-Q_{j j} & -A_{j j}^{T}
\end{array}\right]
$$

Let $\bar{P}_{00}, \bar{P}_{10}, \bar{P}_{20}, \bar{P}_{11}, \bar{P}_{21}$ and $\bar{P}_{22}$ be the limiting solutions of the MARE (5) as $\varepsilon_{j} \rightarrow+0, j=1,2$, $\varepsilon_{12} \rightarrow 0, \varepsilon_{21} \rightarrow 0$, then we obtain the following equations under the assumptions $1-3$.

$$
\begin{aligned}
& A_{s}^{T} \bar{P}_{00}+\bar{P}_{00} A_{s}-\bar{P}_{00} S_{s} \bar{P}_{00}+Q_{s}=0 \\
& \bar{P}_{j 0}^{T}=\bar{P}_{00} N_{0 j}-M_{0 j} \\
& A_{j j}^{T} \bar{P}_{j j}+\bar{P}_{j j} A_{j j}-\bar{P}_{j j} S_{j j} \bar{P}_{j j}+Q_{j j}=0 \\
& \bar{P}_{21}=0
\end{aligned}
$$

$j=1,2$, where

$$
\begin{aligned}
A_{s}:= & A_{00}+N_{01} A_{10}+N_{02} A_{20}+S_{01} M_{01}^{T} \\
& +S_{02} M_{02}^{T}+N_{01} S_{11} M_{01}^{T}+N_{02} S_{22} M_{02}^{T}, \\
S_{s}:= & S_{00}+N_{01} S_{01}^{T}+S_{01} N_{01}^{T}+N_{02} S_{02}^{T} \\
& +S_{02} N_{02}^{T}+N_{01} S_{11} N_{01}^{T}+N_{02} S_{22} N_{02}^{T}, \\
Q_{s}:= & Q_{00}-M_{01} A_{10}-A_{10}^{T} M_{01}^{T}-M_{02} A_{20} \\
& \quad-A_{20}^{T} M_{02}^{T}-M_{01} S_{11} M_{01}^{T}-M_{02} S_{22} M_{02}^{T}, \\
N_{0 j}:= & -D_{0 j} D_{j j}^{-1}, M_{0 j}:=\bar{Q}_{0 j} D_{j j}^{-1}, \\
\bar{Q}_{0 j}:= & A_{j 0}^{T} \bar{P}_{j j}+Q_{0 j}, \\
D_{00}:= & A_{00}-S_{00} \bar{P}_{00}-S_{01} \bar{P}_{10}-S_{02} \bar{P}_{20}, \\
D_{0 j}:= & A_{0 j}-S_{0 j} \bar{P}_{j j}, D_{j j}:=A_{j j}-S_{j j} \bar{P}_{j j}, \\
D_{j 0}:= & A_{j 0}-S_{0 j}^{T} \bar{P}_{00}-S_{j j} \bar{P}_{j 0}, j=1,2 .
\end{aligned}
$$

The matrices $A_{s}, S_{s}$ and $Q_{s}$ do not depend on $\bar{P}_{11}$ and $\bar{P}_{22}$ because their matrices can be computed by using $T_{p q}, p, q=0,1,2$ which are independent of $\bar{P}_{11}$ and $\bar{P}_{22}$ (Coumarbatch and Gajić, 2000), that is,

$$
\begin{aligned}
T_{s} & :=T_{00}-T_{01} T_{11}^{-1} T_{10}-T_{02} T_{22}^{-1} T_{20} \\
& =\left[\begin{array}{cc}
A_{s} & -S_{s} \\
-Q_{s} & -A_{s}^{T}
\end{array}\right], \\
T_{00} & :=\left[\begin{array}{cc}
A_{00} & -S_{00} \\
-Q_{00} & -A_{00}^{T}
\end{array}\right], T_{0 j}:=\left[\begin{array}{cc}
A_{0 j} & -S_{0 j} \\
-Q_{0 j} & -A_{j 0}^{T}
\end{array}\right], \\
T_{j 0} & :=\left[\begin{array}{cc}
A_{j 0} & -S_{0 j}^{T} \\
-Q_{0 j}^{T} & -A_{0 j}^{T}
\end{array}\right], \quad j=1,2 .
\end{aligned}
$$

In the following, we will consider the solution of the reduced-order ARE (9). Before doing that, we first introduce the useful property of the reducedorder ARE (9) (Mukaidani, 2001; Mukaidani and Mizukami, 2001; Mukaidani et al. 2002).

Lemma 1: Under the assumptions 1-3, there exist a matrix $B_{s} \in \mathbf{R}^{n_{0} \times \bar{m}}$ and a matrix $C_{s}$ 
with the same dimension as $\left[C_{10}^{T} C_{20}^{T}\right]^{T}$ such that $S_{s}=B_{s} R^{-1} B_{s}^{T}, Q_{s}=C_{s}^{T} C_{s}$. That is,

$$
\begin{aligned}
& S_{s}:=\left[B_{01}+N_{01} B_{11} B_{02}+N_{02} B_{22}\right] \\
& \cdot\left[\begin{array}{cc}
R_{1}^{-1} & 0 \\
0 & R_{2}^{-1}
\end{array}\right] \cdot\left[\begin{array}{c}
B_{01}^{T}+B_{11}^{T} N_{01}^{T} \\
B_{02}^{T}+B_{22}^{T} N_{02}^{T}
\end{array}\right], \\
& Q_{s}:=\left[C_{10}^{T}+L_{10}^{T} C_{11}^{T} C_{20}^{T}+L_{20}^{T} C_{22}^{T}\right] \\
& \cdot\left[\begin{array}{l}
C_{10}+C_{11} L_{10} \\
C_{20}+C_{22} L_{20}
\end{array}\right]=C_{s}^{T} C_{s}, \\
& j=1,2, L_{j 0}:=-E_{j j}^{-1} E_{j 0}, \\
& E_{j 0}:=A_{j 0}-\tilde{W}_{j j} Q_{0 j}^{T}, E_{j j}:=A_{j j}-\tilde{W}_{j j} Q_{j j}, \\
& \tilde{W}_{j j} A_{j j}^{T}+A_{j j} \tilde{W}_{j j}-\tilde{W}_{j j} Q_{j j} \tilde{W}_{j j}+S_{j j}=0 \text {, }
\end{aligned}
$$

Moreover, the triple $\left(A_{s}, B_{s}, C_{s}\right)$ is stabilizable and detectable.

It should be remarked that the solution $P_{e}$ of the MARE (5) is a function of the multiparameters $\varepsilon_{j}, j=1,2, \varepsilon_{12}$ and $\varepsilon_{21}$. But, the solutions $\bar{P}_{00}$, $\bar{P}_{11}$ and $\bar{P}_{22}$ of $(9 \mathrm{a})$ and $(9 \mathrm{c})$ are independent of the multiparameters $\varepsilon_{j}, \varepsilon_{12}$ and $\varepsilon_{21}$, respectively. Moreover, we do not assume here that $A_{j j}, j=$ 1,2 are nonsingular. Thus, our new results are applicable to more realistic MSPS compared with the existing results (Gajić, 1988).

The following lemma will establish the relation between $P_{e}$ and the reduced-order solutions (9) (Mukaidani, 2001; Mukaidani and Mizukami, 2001; Mukaidani et al. 2002).

Lemma 2: Under the assumptions 1-3, there exists small $\sigma^{*}$ such that for all $\|\mu\| \in\left(0, \sigma^{*}\right)$ the MARE (5) admits a symmetric positive semidefinite stabilizing solution $P_{e}$ which can be written as

$$
\begin{aligned}
& P_{e}=\left[\begin{array}{cc}
\bar{P}_{00}+O(\|\mu\|) & \varepsilon_{1}\left(\bar{P}_{10}+O(\|\mu\|)\right)^{T} \\
\varepsilon_{1}\left(\bar{P}_{10}+O(\|\mu\|)\right) & \varepsilon_{1}\left(\bar{P}_{11}+O(\|\mu\|)\right) \\
\varepsilon_{2}\left(\bar{P}_{20}+O(\|\mu\|)\right) & \sqrt{\varepsilon_{1} \varepsilon_{2}} O(\|\mu\|)
\end{array}\right. \\
& \left.\begin{array}{c}
\varepsilon_{2}\left(\bar{P}_{20}+O(\|\mu\|)\right)^{T} \\
\sqrt{\varepsilon_{1} \varepsilon_{2}} O(\|\mu\|) \\
\varepsilon_{2}\left(\bar{P}_{22}+O(\|\mu\|)\right)
\end{array}\right] .
\end{aligned}
$$

It should be noted that the entries $(2,3)$ and $(3,2)$ of the solution $P_{e}$ can be written as $\sqrt{\varepsilon_{1} \varepsilon_{2}} O(\|\mu\|)$ because $\bar{P}_{21}=0$. With respect to the results of Gajić (1988), we do not require the singularity of $A_{j j}, j=1,2$.

\section{NEAR-OPTIMAL CONTROL}

The required solution of the MARE (5) exists under the assumptions 1-3. Our attention is focused on the specific linear state feedback controller which does not depend on the values of the small parameters. Such the linear state feedback controller is obtained by eliminating $O(\|\mu\|)$ item of the linear state feedback controller (4). If $\|\mu\|:=\sqrt{\varepsilon_{1} \varepsilon_{2}}$ is very small, it is obvious that the linear state feedback controller (4) can be approximated as

$$
\begin{aligned}
u_{\mathrm{app}}(t) & =-R^{-1} B^{T} \bar{P} x(t) \\
& =-R^{-1} B^{T}\left[\begin{array}{ccc}
\bar{P}_{00} & 0 & 0 \\
\bar{P}_{10} & \bar{P}_{11} & 0 \\
\bar{P}_{20} & 0 & \bar{P}_{22}
\end{array}\right] x(t),(12)
\end{aligned}
$$

where $B=\Phi_{e} B_{e}$,

$\Phi_{e}=$ block $-\operatorname{diag}\left(\begin{array}{lll}I_{n_{0}} & \varepsilon_{1} I_{n_{1}} & \varepsilon_{2} I_{n_{2}}\end{array}\right)$.

Remark: Even though it is quite different from the composite controller design (Khalil and Kokotović, 1979; Wang et al., 1994;Xu et al., 1997), the resulting controller (12) is similar to the existing one. In fact, it will be proved later.

When $\|\mu\|$ is sufficiently small, we know from Lemma 2 that the resulting controller (12) will be close to the optimal controller (4). In an optimization problem it is of interest to check whether the resulting value of the cost function will be near its optimal value.

Theorem 1: Under the assumptions 1-3, the use of the reduced-order controller (12) results in $J_{\text {app }}$ satisfying

$$
J_{\mathrm{app}}=J_{\mathrm{opt}}+O\left(\|\mu\|^{2}\right)
$$

Proof: When $u_{\text {app }}$ is used, the value of the performance index is

$$
J_{\mathrm{app}}=\frac{1}{2} x(0)^{T} W_{e} x(0)
$$

where $W_{e}$ is a positive semidefinite solution of the following maltiparameter algebraic Lyapunov equation (MALE) as $\bar{P}_{e}=\Phi_{e} \bar{P}$

$$
\begin{aligned}
& \left(A_{e}-S_{e} \bar{P}_{e}\right)^{T} W_{e}+W_{e}\left(A_{e}-S_{e} \bar{P}_{e}\right) \\
& \quad+\bar{P}_{e} S_{e} \bar{P}_{e}+Q=0 .
\end{aligned}
$$

Subtracting (5) from (15) we find that $V_{e}=W_{e}-$ $P_{e}$ satisfies the following MALE

$$
\begin{gathered}
\left(A_{e}-S_{e} \bar{P}_{e}\right)^{T} V_{e}+V_{e}\left(A_{e}-S_{e} \bar{P}_{e}\right) \\
+\left(P_{e}-\bar{P}_{e}\right) S_{e}\left(P_{e}-\bar{P}_{e}\right)=0
\end{gathered}
$$

We again assume the form (17) for $V_{e}$ as follows.

$$
V_{e}=\left[\begin{array}{ccc}
V_{00} & \varepsilon_{1} V_{10}^{T} & \varepsilon_{2} V_{20}^{T} \\
\varepsilon_{1} V_{10} & \varepsilon_{1} V_{11} & \sqrt{\varepsilon_{1} \varepsilon_{2}} V_{21}^{T} \\
\varepsilon_{2} V_{20} & \sqrt{\varepsilon_{1} \varepsilon_{2}} V_{21} & \varepsilon_{2} V_{22}
\end{array}\right]
$$

The MALE (16) can be partitioned into 


$$
\begin{aligned}
& D_{00}^{T} V_{00}+V_{00} D_{00}+D_{10}^{T} V_{10}+V_{10}^{T} D_{10}+D_{20}^{T} V_{20} \\
& \quad+V_{20}^{T} D_{20}+O\left(\|\mu\|^{2}\right)=0, \\
& V_{00} D_{01}+V_{10}^{T} D_{11}+\varepsilon_{21} V_{20}^{T} A_{21}+\varepsilon_{1} D_{00}^{T} V_{10}^{T} \\
& \quad+D_{10}^{T} V_{11}+\sqrt{\alpha} D_{20}^{T} V_{21}+O\left(\|\mu\|^{2}\right)=0, \quad(18 \mathrm{~b}) \\
& V_{00} D_{02}+V_{20}^{T} D_{22}+\varepsilon_{12} V_{10}^{T} A_{12}+\varepsilon_{2} D_{00}^{T} V_{20}^{T} \\
& \quad+D_{20}^{T} V_{22}+\frac{1}{\sqrt{\alpha}} D_{10}^{T} V_{21}^{T}+O\left(\|\mu\|^{2}\right)=0, \quad(18 \mathrm{c}) \\
& D_{11}^{T} V_{11}+V_{11} D_{11}+\varepsilon_{1}\left(D_{01}^{T} V_{10}^{T}+V_{10} D_{01}\right) \\
& \quad+\sqrt{\alpha} \varepsilon_{21}\left(V_{21}^{T} A_{21}+A_{21}^{T} V_{21}\right)+O\left(\|\mu\|^{2}\right)=0,(18 \mathrm{~d}) \\
& \varepsilon_{1} V_{10} D_{02}+\varepsilon_{2} D_{01}^{T} V_{20}^{T}+\varepsilon_{12} V_{11} A_{12}+\varepsilon_{21} A_{21}^{T} V_{22} \\
& \quad+\sqrt{\alpha} V_{21}^{T} D_{22}+\frac{1}{\sqrt{\alpha}} D_{11}^{T} V_{21}^{T}+O\left(\|\mu\|^{2}\right)=0,(18 \mathrm{e}) \\
& D_{22}^{T} V_{22}+V_{22} D_{22}+\varepsilon_{2}\left(D_{02}^{T} V_{20}^{T}+V_{20} D_{02}\right) \\
& \quad+\frac{1}{\sqrt{\alpha}} \varepsilon_{12}\left(V_{21} A_{12}+A_{12}^{T} V_{21}^{T}\right)+O\left(\|\mu\|^{2}\right)=0,(18 \mathrm{f})
\end{aligned}
$$

where

$$
\begin{gathered}
A_{e}-S_{e} \bar{P}_{e}=\Phi_{e}^{-1}\left[\begin{array}{ccc}
D_{00} & D_{01} & D_{02} \\
D_{10} & D_{11} & \varepsilon_{12} A_{12} \\
D_{20} & \varepsilon_{21} A_{21} & D_{22}
\end{array}\right], \\
\left(P_{e}-\bar{P}_{e}\right) S_{e}\left(P_{e}-\bar{P}_{e}\right)=O\left(\|\mu\|^{2}\right) .
\end{gathered}
$$

Setting $\|\mu\|=0$ for the above equations (18), we have

$$
\begin{aligned}
& D_{00}^{T} \bar{V}_{00}+\bar{V}_{00} D_{00}+D_{10}^{T} \bar{V}_{10}+\bar{V}_{10}^{T} D_{10} \\
& \quad+D_{20}^{T} \bar{V}_{20}+\bar{V}_{20}^{T} D_{20}=0 \\
& \bar{V}_{00} D_{01}+\bar{V}_{10}^{T} D_{11}+D_{10}^{T} \bar{V}_{11}+\sqrt{\bar{\alpha}} D_{20}^{T} \bar{V}_{21}=0 \\
& \bar{V}_{00} D_{02}+\bar{V}_{20}^{T} D_{22}+D_{20}^{T} \bar{V}_{22}+\frac{1}{\sqrt{\bar{\alpha}}} D_{10}^{T} \bar{V}_{21}^{T}=0 \\
& D_{j j}^{T} \bar{V}_{j j}+\bar{V}_{j j} D_{j j}=0, j=1,2 \\
& \sqrt{\bar{\alpha}} \bar{V}_{21}^{T} D_{22}+\frac{1}{\sqrt{\bar{\alpha}}} D_{11}^{T} \bar{V}_{21}^{T}=0 .
\end{aligned}
$$

Since $D_{11}$ and $D_{22}$ are stable, using the standard properties of the algebraic Lyapunov equation (ALE) (Zhou, 1998), we deduce that $\bar{V}_{p q}=$ $0, p q=11,21,22$, where $\bar{V}_{p q}=0$ are 0 -order solutions of (18). Using $\bar{V}_{p q}=0, p q=11,21,22$, we get

$$
\begin{aligned}
& D_{0}^{T} \bar{V}_{00}+\bar{V}_{00} D_{0}=0, \\
& \bar{V}_{j 0}^{T}=-\bar{V}_{00} D_{0 j} D_{j j}^{-1}, j=1,2 .
\end{aligned}
$$

Since $D_{0}:=A_{s}-S_{s} \bar{P}_{00}$ is stable from the ARE (9a) (Mukaidani, 2001; Mukaidani et al. 2002), we also deduce that $\bar{V}_{p q}=0, p q=00,10,20$. Thus, we have

$V_{p q}=O(\|\mu\|) V_{p q}^{(1)}, p q=00,10,20,11,21,22$.

Substituting $V_{p q}=O(\|\mu\|) V_{p q}^{(1)}$ into (18) and by following the above steps, we obtain the following equations because $\bar{V}_{p q}^{(1)}=\left.V_{p q}^{(1)}\right|_{\|\mu\|=0}=0$.

$$
V_{p q}=O\left(\|\mu\|^{2}\right) V_{p q}^{(2)}, V_{p q}^{(2)} \neq 0 .
$$

Hence

$$
V_{e}=W_{e}-P_{e}=O\left(\|\mu\|^{2}\right),
$$

which implies (13).

We have therefore provided a complete theoretic analysis of the near-optimality of the reducedorder optimal control for the MSPS. Note that the intuitive result of Theorem 1 can be also proved by using Newton-Kantorovich theorem (Ortega, 1970).

In the rest of this section, we will show that the near-optimal controller (12) is equivalent to the existing composite optimal controller (Wang et al., 1994;Kokotović et al., 1986). Let $A_{j j}, j=$ 1,2 of (1) be nonsingular. Then, the composite optimal controller is

$$
\begin{aligned}
u_{\mathrm{com}}(t) & =-R^{-1} B^{T} X x(t) \\
& =-R^{-1} B^{T}\left[\begin{array}{ccc}
X_{00} & 0 & 0 \\
X_{10} & X_{11} & 0 \\
X_{20} & 0 & X_{22}
\end{array}\right] x(t) .
\end{aligned}
$$

In the above, $X_{00}$ is the unique stabilizing positive semidefinite symmetric solution of the ARE

$$
\begin{aligned}
& \left(A_{r}-B_{r} R_{r}^{-1} E_{r}^{T} C_{r}\right)^{T} X_{00} \\
& \quad+X_{00}\left(A_{r}-B_{r} R_{r}^{-1} E_{r}^{T} C_{r}\right)-X_{00} B_{r} R_{r}^{-1} B_{r}^{T} X_{00} \\
& \quad+C_{r}^{T}\left(I_{n_{0}}-E_{r} R_{r}^{-1} E_{r}^{T}\right) C_{r}=0,
\end{aligned}
$$

where

$$
\begin{aligned}
& A_{r}=A_{00}-A_{01} A_{11}^{-1} A_{10}-A_{02} A_{22}^{-1} A_{20}, \\
& B_{r}=\left[\begin{array}{ll}
B_{01}-A_{01} A_{11}^{-1} B_{11} B_{02}-A_{02} A_{22}^{-1} B_{22}
\end{array}\right], \\
& C_{r}=\left[\begin{array}{c}
C_{10}-C_{11} A_{11}^{-1} A_{10} \\
C_{20}-C_{22} A_{22}^{-1} A_{20}
\end{array}\right], R_{r}=R+E_{r}^{T} E_{r}, \\
& E_{r}=-\left[\begin{array}{cc}
C_{11} A_{11}^{-1} B_{11} & 0 \\
0 & C_{22} A_{22}^{-1} B_{22}
\end{array}\right] .
\end{aligned}
$$

$X_{j j}, j=1,2$ are the unique stabilizing positive semidefinite solution of the following AREs

$$
A_{j j}^{T} X_{j j}+X_{j j} A_{j j}-X_{j j} S_{j j} X_{j j}+Q_{j j}=0,
$$

and $X_{j 0}, j=1,2$ are

$$
\begin{gathered}
X_{j 0}^{T}=\left[X_{00}\left(S_{0 j} X_{j j}-A_{0 j}\right)-\left(A_{j 0}^{T} X_{j j}\right.\right. \\
\left.\left.+Q_{0 j}\right)\right]\left(A_{j j}-S_{j j} X_{j j}\right)^{-1} .
\end{gathered}
$$

Theorem 2: Suppose that the fast state matrices $A_{j j}, j=1,2$ are nonsingular. Under the assumption $1-3$, the following identities

$X_{j j}=\bar{P}_{j j}, X_{j 0}=\bar{P}_{j 0}, X_{00}=\bar{P}_{00}, j=1,2$, 
hold. Hence the resulting near--optimal controller (12) is the same as the composite optimal controller (21).

Proof: First, comparing (23) with (9c) $X_{j j}=$ $\bar{P}_{j j}, j=1,2$ yields directly. Second, comparing (24) with (9b) and noting that $X_{j j}=\bar{P}_{j j}$, we have the conclusion that $X_{j 0}=\bar{P}_{j 0}, j=1,2$ if $X_{00}=\bar{P}_{00}$. Therefore, the remainder of the proof is to show that $X_{00}=\bar{P}_{00}$. In order to do that, we only need to show that

$$
\begin{aligned}
& A_{r}-B_{r} R_{r}^{-1} E_{r}^{T} C_{r}=A_{s}, \\
& B_{r} R_{r}^{-1} B_{r}^{T}=S_{s}, \\
& C_{r}^{T}\left(I_{n_{0}}-E_{r} R_{r}^{-1} E_{r}^{T}\right) C_{r}=Q_{s} .
\end{aligned}
$$

Before showing these relations, let us define (pp.115, Kokotović et al. 1986)

$$
\begin{aligned}
& H=I_{n_{1}+n_{2}} \\
& +\left[\begin{array}{cc}
R_{1}^{-1} B_{11}^{T} \bar{P}_{11} D_{11}^{-1} B_{11} & 0 \\
0 & R_{2}^{-1} B_{22}^{T} \bar{P}_{22} D_{22}^{-1} B_{22}
\end{array}\right](27)
\end{aligned}
$$

Then,

$$
\begin{aligned}
& H^{-1}=I_{n_{1}+n_{2}} \\
& -\left[\begin{array}{cc}
R_{1}^{-1} B_{11}^{T} \bar{P}_{11} A_{11}^{-1} B_{11} & 0 \\
0 & R_{2}^{-1} B_{22}^{T} \bar{P}_{22} A_{22}^{-1} B_{22}
\end{array}\right]
\end{aligned}
$$

Thus, using (28) and the ARE (9c) we have

$$
H R^{-1} H^{T}=R_{r}^{-1} .
$$

Let us further introduce six useful identities.

$$
\begin{aligned}
& A_{j j}^{-1}+A_{j j}^{-1} S_{j j} \bar{P}_{j j} D_{j j}^{-1}=D_{j j}^{-1}, \\
& A_{j j}^{-1}+D_{j j}^{-1} S_{j j} \bar{P}_{j j} A_{j j}^{-1}=D_{j j}^{-1}, \\
& I_{n_{j}}+S_{j j} \bar{P}_{j j} D_{j j}^{-1}=A_{j j} D_{j j}^{-1}, \\
& I_{n_{j}}+\bar{P}_{j j} S_{j j} D_{j j}^{-T}=A_{j j}^{T} D_{j j}^{-T}, \\
& Q_{0 j}^{T}-Q_{j j} A_{j j}^{-1} A_{j 0}=\bar{Q}_{0 j}^{T}+D_{j j}^{T} \bar{P}_{j j} A_{j j}^{-1} A_{j 0}, \\
& -D_{0 j}+N_{0 j} S_{j j} \bar{P}_{j j}=N_{0 j} A_{j j}, j=1,2 .
\end{aligned}
$$

Hence, using the above relation, we have $A_{r}-$ $B_{r} R_{r}^{-1} E_{r}^{T} C_{r}=A_{s}$ which proves (26a). Due to the page limitation, the rest of the proof of Theorem 2 is omitted. (See for e.g., Xu et al., 1999; Mukaidani, 2001)

From Theorem 2, we claim that the new nearoptimal controller includes the existing composite optimal controller (21) as the special case.

\section{CONCLUSION}

In this paper, the optimal control problem associated with the MSPS has been considered. The main contribution of this paper is to propose the new design method of the $\varepsilon_{j}$-independent reduced-order controller. Note that the proposed design method is quite different from the existing method such as the two-time-scale design method and the descriptor variable approach. It has been newly shown that the resulting controller achieves $O\left(\|\mu\|^{2}\right)$ approximation of the optimal cost. Furthermore, since it has been proven that the resulting controller is equivalent to the existing composite controller, when the fast subsystems have the special form such controllers will achieve the $O\left(\|\mu\|^{2}\right)$ approximation.

\section{References}

Coumarbatch, C. and Z. Gajić (2000). Exact decomposition of the algebraic Riccati equation of deterministic multimodeling optimal control problems. IEEE Trans. A.C, 45, 790-794.

Gajić, Z. (1988). The existence of a unique and bounded solution of the algebraic Riccati equation of multimodel estimation and control problems. Sys. \& Con. Lett., 10, 185-190.

Khalil, H. K. and P. V. Kokotović (1978). Control strategies for decision makers using different models of the same system. IEEE Trans. A.C., 23, 289-298.

Khalil, H. K. and P. V. Kokotović (1979). Control of linear systems with multiparameter singular perturbations. Automatica, 15, 197-207.

Kokotović, P. V., H. K. Khalil and J. O'Reilly (1986). Singular perturbation methods in control: analysis and design. New York: Academic Press.

Mukaidani, H. (2001). Pareto near-optimal strategy of multimodeling systems. In Proc. IEEE Int. Conf. IECON01, Denver, 500-505.

Mukaidani, H. and K. Mizukami (2001). Control of linear multiparameter singularity pertubed systems, In Proc. IFAC Workshop on Singular Solutions and Perturbations, Bucharest, 13-18. Mukaidani, H., H. Xu and K. Mizukami (2002). Recursive computation of pareto optimal strategy for multiparameter singularly perturbed systems. Dyn. Continuous, Discrete and Impulsive Systems, 9b, 175-200.

Ortega, J. M. and W. C. Rheinboldt (1970). Iterative Solution of Nonlinear Equations in Several Variables. New York: Academic Press. Wang, Y-Y., M. Paul and N. E. Wu (1994). Near-optimal control of nonstandard singularly perturbed systems. Automatica, 30, 277-292.

Xu, H., H. Mukaidani and K. Mizukami (1997). : New Method for Composite Optimal Control of Singularly Perturbed Systems, International. Int. J. Systems Sciences, 28, 161-172.

Zhou, K. (1998). Essentials of Robust Control. New Jersey: Prentice-Hall. 